\title{
Mechanical properties and experimental researches of new CSIPs sandwich panels
}

\author{
Wenfeng Du, Xue Yang, Liming Zhu ${ }^{*}$ and Yun Sun \\ Institute of Steel and Spatial Structures in School of Civil Engineering and Architecture, Henan University, Kaifeng \\ 475004 China \\ *Corresponding author: dwf@henu.edu.cn
}

\begin{abstract}
The advantages of glass fiber reinforced composites (FRP) and SIPs (structural insulated panels) are combined, and a new type of sandwich panel called composite structural insulated panels (CSIPs) is proposed. Through the adhesive bonding, CSIPs are made of FRP as face sheets and expanded polyethylene foam (EPS) as a core. To master the mechanical characteristics of CSIPs, firstly, adopting the large deflection theory of Reissener in this paper derived the calculation formula of displacement and the stability critical load of CSIPs. Then, ANSYS software was used to carry on the analysis of finite element simulation. Finally, a testing piece of CSIP with length $1000 \mathrm{~mm}$ and breath $1000 \mathrm{~mm}$ was made and a test was done. The results show that the theoretical analysis results, finite element simulation results and test results are basically coincide. So the calculating formula of deformation and bearing capacity of CSIPs are correct. And CSIPs have the outstanding advantages of light weight and high strength.
\end{abstract}

\section{Introduction}

The earliest sandwich construction originated from the corrugated board structure invented by the British in 1856 . It adds a corrugated paper core between the two layers of paper to make the soft paper to obtain a certain stiffness[1].In 1935, a forest products laboratory (FPL) in the United States proposed sandwich panel structures, namely SIPs (Structural Insulated Panels), which is composed of two layers of the Oriented Strand Board (OSB) as face sheets and a layer of thermal insulation laminated composite panel as a core[2-3].Currently SIPs have been extensively used all over the world, however, the SIPs have the disadvantages of poor fire resistance, easy occurrence of pest and warping deformation.

In recent years, application of FRP (Fiber Reinforced Plastic Composites) in civil engineering structure developed rapidly. The FRP is comprised of enhanced fiber material and matrix material through unique moulding process with the advantages of light weight, high strength, corrosion resistant, the ability of customize, durability, energy conservation and environmental protection and so on. Based on the traditional SIPs, a new kind of sandwich panel structure, namely CSIPs (Composite Structural Insulated Panels), was proposed by this paper using the FRP instead of the OSB. CSIPs have inherited sandwich structural characteristic and production process of SIPs, at the same time, effectively overcome the disadvantages of poor fire resistance, easy occurrence of pest and warping deformation. Moreover, because the strength of FRP is much higher than wood, CSIPs can improve mechanical properties of SIPs greatly.

CSIPs are made of low-cost orthotropic thermoplastic glass/polypropylene (glass-PP) laminate as face sheets and expanded polyethylene (EPS) foam as a core through a hot-melt thermoplastic spray adhesive bonding together. GlassPP laminates provide high strength-to-weight ratio, excellent impact resistance, and high durability. EPS, on the other hand, are marked by light weight, thermal insulation, good fire resistance and excellent impact properties. All these features lead finally to superior performance of the panels. Therefore, CSIPs have broad application prospects in the wall and roof structures [4-6].

In order to provide a good foundation for the application of CSIPs, the mechanistic properties of CSIPs were studied by means of theoretical analysis, finite element simulation and experimental study.

\section{Theoretical analysis}

\subsection{Basic assumptions}

In this paper, the calculation formulas of internal force and displacement in sandwich panels are derived from Reissner theory, which takes into account the basic high-order partial differential equation for the sandwich plate with large deflection bending. The following assumptions are advanced on CSIPs [7-8]: 
(1) For the thickness of the surface layer is very small relatively comparing to the whole sandwich panel, a thin film was used to simulate face sheets. That is assuming that the surface layer is in the state of film stress;

(2) Due to the core is soft, it can be ignored that force components of sandwich panel in parallel the XY plane, this is assuming that $\sigma_{\mathrm{x}}=\sigma_{\mathrm{y}}=\sigma_{\mathrm{xy}}=0$;

(3) It is assumed that the strain in the core and the surface layer along the thickness direction is zero $\left(\varepsilon_{z}=0\right)$;

(4) The stress $\sigma_{z}$ along the thickness direction is very small;

(5) The transverse shear effect was considered and the straight line segment along the direction of thickness keeps a straight line after the deformation even though it is not perpendicular to the middle plane.

\subsection{The displacement and stress components of the sandwich panel}

The displacements of any point in the sandwich panel are as following:

The upper face sheet

$$
u_{1}=u+\frac{1}{2} c \varphi_{x} \quad v_{1}=v+\frac{1}{2} c \varphi_{y} \quad w_{1}=w
$$

The core

$$
u_{2}=u+z \varphi_{x} \quad v_{2}=v+z \varphi_{y} \quad w_{2}=w
$$

The lower face sheet

$$
u_{3}=u-\frac{1}{2} c \varphi_{x} \quad v_{3}=v-\frac{1}{2} c \varphi_{y} \quad w_{3}=w
$$

In the above equations, $u_{i}, v_{i}, w_{i}$ are the displacement components of the upper, the lower face sheet and the core. $\varphi_{\mathrm{x}}(\mathrm{x}, \mathrm{y})$ and $\varphi_{\mathrm{y}}(\mathrm{x}, \mathrm{y})$ are rotation angles in the $\mathrm{XY}$ and $\mathrm{YZ}$ plane.

The geometric equations of sandwich panel are as following:

$$
\begin{aligned}
& \varepsilon_{x \mathrm{i}}=\frac{\partial u_{i}}{\partial x}+\frac{1}{2}\left(\frac{\partial w_{i}}{\partial x}\right)^{2} \\
& \varepsilon_{y \mathrm{i}}=\frac{\partial v_{i}}{\partial y}+\frac{1}{2}\left(\frac{\partial w_{i}}{\partial y}\right)^{2} \\
& \gamma_{x y \mathrm{i}}=\frac{\partial v_{i}}{\partial x}+\frac{\partial u_{i}}{\partial y}+\frac{\partial w_{i}}{\partial x} \frac{\partial w_{i}}{\partial y} \quad(i=1,3) \\
& \varepsilon_{z \mathrm{i}}=\gamma_{x z \mathrm{i}}=\gamma_{y z \mathrm{i}}=0 \\
& \gamma_{x z 2}=\frac{\partial u_{2}}{\partial z}+\frac{\partial w_{2}}{\partial x} \\
& \gamma_{y z 2}=\frac{\partial v_{2}}{\partial z}+\frac{\partial w_{2}}{\partial y} \\
& \varepsilon_{x 2}=\varepsilon_{y 2}=\varepsilon_{z 2}=\gamma_{x y 2}=0
\end{aligned}
$$

In the above equations $\varepsilon_{x i}, \varepsilon_{y i}, \varepsilon_{z i}, \gamma_{x y i}, \gamma_{y z i}$ and $\gamma_{x z i}(\mathrm{i}=1,2,3)$ are the strain components of the upper, the lower face sheet and the core.

The strain components of the upper face sheet

The strain components of the lower face sheet

$$
\begin{aligned}
& \sigma_{x 1}=\sigma_{x 0}+\frac{E_{f} c}{2\left(1-v_{f}^{2}\right)}\left(\frac{\partial \varphi_{x}}{\partial x}+v_{f} \frac{\partial \varphi_{y}}{\partial y}\right) \\
& \sigma_{y 1}=\sigma_{y 0}+\frac{E_{f} c}{2\left(1-v_{f}^{2}\right)}\left(\frac{\partial \varphi_{y}}{\partial y}+v_{f} \frac{\partial \varphi_{x}}{\partial x}\right) \\
& \tau_{x y 1}=\tau_{x y 0}+\frac{G c}{2}\left(\frac{\partial \varphi_{y}}{\partial x}+\frac{\partial \varphi_{x}}{\partial y}\right)
\end{aligned}
$$


The strain components of the core

$$
\begin{aligned}
& \sigma_{x 3}=\sigma_{x 0}-\frac{E_{f} c}{2\left(1-v_{f}^{2}\right)}\left(\frac{\partial \varphi_{x}}{\partial x}+v_{f} \frac{\partial \varphi_{y}}{\partial y}\right) \\
& \sigma_{y 3}=\sigma_{y 0}-\frac{E_{f} c}{2\left(1-v_{f}^{2}\right)}\left(\frac{\partial \varphi_{y}}{\partial y}+v_{f} \frac{\partial \varphi_{x}}{\partial x}\right) \\
& \tau_{x y 3}=\tau_{x y 0}-\frac{G c}{2}\left(\frac{\partial \varphi_{y}}{\partial x}+\frac{\partial \varphi_{x}}{\partial y}\right)
\end{aligned}
$$

$$
\begin{aligned}
& \tau_{x z 2}=G_{\text {core }}\left(\varphi_{x}+\frac{\partial w}{\partial x}\right) \\
& \tau_{y z 2}=G_{\text {core }}\left(\varphi_{y}+\frac{\partial w}{\partial y}\right)
\end{aligned}
$$

Where $\varepsilon_{x i}, \varepsilon_{y i}, \varepsilon_{z i}, \varepsilon_{x y i}, \varepsilon_{y z i}$ and $\varepsilon_{x z i}(\mathrm{i}=1,2,3)$ are the strain components of the upper, the lower face sheet and the core. $E_{f}$ and $v_{f}$ are elastic moduli and poisson's ratios. $G$ and $G_{c o r e}$ are the shearing modulus of the face sheet core.

$$
\begin{aligned}
& \sigma_{x 0}=\frac{E_{f}}{\left(1-v_{f}^{2}\right)}\left[\frac{\partial u}{\partial x}+v_{f} \frac{\partial v}{\partial y}+\frac{1}{2}\left(\frac{\partial w}{\partial x}\right)^{2}+\frac{v_{f}}{2}\left(\frac{\partial w}{\partial y}\right)^{2}\right] \\
& \sigma_{y 0}=\frac{E_{f}}{\left(1-v_{f}^{2}\right)}\left[\frac{\partial v}{\partial y}+v_{f} \frac{\partial u}{\partial x}+\frac{1}{2}\left(\frac{\partial w}{\partial y}\right)^{2}+\frac{v_{f}}{2}\left(\frac{\partial w}{\partial x}\right)^{2}\right](8) \\
& \tau_{x y 0}=G\left(\frac{\partial v}{\partial x}+\frac{\partial u}{\partial y}+\frac{\partial w}{\partial x} \frac{\partial w}{\partial y}\right)
\end{aligned}
$$

Where $\sigma_{x 0}, \sigma_{y 0}$ and $\tau_{x y 0}$ are the strains of intermediate surface in sandwich panel.

\subsection{General bending moment and shear in sandwich panel}

Because the transverse equilibrium equations are the same as those of the single layer panel, the bending moment, the torque and the shear force in sandwich panel can be obtained:

$$
\begin{aligned}
& M_{x}=-D\left(\frac{\partial \varphi_{x}}{\partial x}+v_{f} \frac{\partial \varphi_{y}}{\partial y}\right) \\
& M_{y}=-D\left(\frac{\partial \varphi_{y}}{\partial y}+v_{f} \frac{\partial \varphi_{x}}{\partial x}\right) \\
& M_{x y}=\frac{D}{2}\left(1-v_{f}\right)\left(\frac{\partial \varphi_{x}}{\partial y}+\frac{\partial \varphi_{y}}{\partial x}\right) \\
& Q_{x}=G_{\text {core }} c\left(\varphi_{x}+\frac{\partial w}{\partial x}\right) \\
& Q_{y}=G_{\text {core }} c\left(\varphi_{y}+\frac{\partial w}{\partial y}\right)
\end{aligned}
$$

Where $D$ is the flexural rigidity of the sandwich panel.

\subsection{The fundamental equations and boundary conditions}

According to the elastic strain energy formula, the total potential energy of the sandwich panel can be obtained: 
So according to potential energy principle:

$$
\begin{aligned}
& U=\frac{t}{E} \iint_{s}\left[\begin{array}{l}
\left(\sigma_{x 0}+\sigma_{y 0}\right)^{2}+ \\
2\left(1+v_{f}\right)\left(\tau_{x y 0}^{2}-\sigma_{x 0} \sigma_{y 0}\right)
\end{array}\right] d x d y \\
& +\frac{D}{2} \iint_{s}\left[\begin{array}{l}
\left(\frac{\partial \varphi_{x}}{\partial x}\right)^{2}+\left(\frac{\partial \varphi_{y}}{\partial y}\right)^{2}+ \\
2 v_{f} \frac{\partial \varphi_{x}}{\partial x} \frac{\partial \varphi_{y}}{\partial y}+\frac{1-v_{f}}{2}\left(\frac{\partial \varphi_{y}}{\partial x}+\frac{\partial \varphi_{x}}{\partial y}\right)^{2}
\end{array}\right] d x d y
\end{aligned}
$$

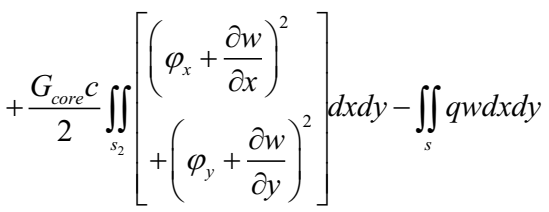

$$
\begin{aligned}
& \delta U=0 \\
& \frac{D}{G_{\text {core }} c}\left(\frac{\partial^{2} \varphi_{x}}{\partial x^{2}}+\frac{1-v_{f}}{2} \frac{\partial^{2} \varphi_{y}}{\partial y^{2}}+\frac{1+v_{f}}{2} \frac{\partial^{2} \varphi_{y}}{\partial x \partial y}\right) \\
& -\left(\frac{\partial w}{\partial x}+\varphi_{x}\right)=0 \\
& \frac{D}{G_{\text {core }} c}\left(\frac{\partial^{2} \varphi_{y}}{\partial y^{2}}+\frac{1-v_{f}}{2} \frac{\partial^{2} \varphi_{y}}{\partial x^{2}}+\frac{1+v_{f}}{2} \frac{\partial^{2} \varphi_{x}}{\partial x \partial y}\right) \\
& -\left(\frac{\partial w}{\partial y}+\varphi_{y}\right)=0 \\
& G_{\text {core }} c\left(\frac{\partial \varphi_{x}}{\partial x}+\frac{\partial \varphi_{y}}{\partial y}+\nabla^{2} w\right) \\
& =-q-2 t\left(\sigma_{x 0} \frac{\partial^{2} w}{\partial x^{2}}+\sigma_{y 0} \frac{\partial^{2} w}{\partial y^{2}}+2 \tau_{x y 0} \frac{\partial^{2} w}{\partial x \partial y}\right)
\end{aligned}
$$

The boundary conditions

$$
\begin{gathered}
\text { when } x=0, a \\
\sigma_{x 0}=0 \quad \text { or } \quad \delta u=0 \\
\tau_{x y 0}=0 \quad \text { or } \quad \delta v=0 ; \\
G_{\text {core }} c\left(\varphi_{x}+\frac{\partial w}{\partial x}\right)+2 t\left(\sigma_{x 0} \frac{\partial w}{\partial x}+\tau_{x y 0} \frac{\partial w}{\partial y}\right)=0 \\
\text { or } \quad \delta w=0 ; \\
\frac{D\left(\frac{\partial \varphi_{x}}{\partial x}+v_{f} \frac{\partial \varphi_{y}}{\partial y}\right)=0 \quad \text { or } \quad \delta \varphi_{x}=0 ;}{2}\left(\frac{\partial \varphi_{y}}{\partial x}+\frac{\partial \varphi_{x}}{\partial y}\right)=0 \quad \text { or } \quad \delta \varphi_{y}=0 \\
\text { when } y= \pm \frac{b}{2}
\end{gathered}
$$




$$
\begin{aligned}
& \tau_{x y 0}=0 \quad \text { or } \quad \delta u=0 \\
& \sigma_{y 0}=0 \quad \text { or } \quad \delta v=0 ; \\
& G_{c o r e} c\left(\varphi_{y}+\frac{\partial w}{\partial y}\right)+2 t\left(\frac{\partial w}{\partial x}+\sigma_{y 0} \frac{\partial w}{\partial y}\right)=0 \\
& \text { or } \delta w=0 ; \\
& D\left(\frac{\partial \varphi_{y}}{\partial y}+v_{f} \frac{\partial \varphi_{x}}{\partial x}\right)=0 \quad \text { or } \quad \delta \varphi_{y}=0 ; \\
& \frac{D\left(1-v_{f}\right)}{2}\left(\frac{\partial \varphi_{y}}{\partial x}+\frac{\partial \varphi_{x}}{\partial y}\right)=0 \quad \text { or } \quad \delta \varphi_{x}=0
\end{aligned}
$$

Then according to Hu Haichang's methods which cope with the small deflection equations sandwich panel, $\varphi x, \varphi y$ can be express using the new function $\omega, f[9]$ :

We can get:

$$
\begin{gathered}
\varphi_{x}=\frac{\partial \omega}{\partial x}+\frac{\partial f}{\partial y} \\
\varphi_{y}=\frac{\partial \omega}{\partial y}-\frac{\partial f}{\partial x}
\end{gathered}
$$

$\omega, f$ can be regarded as two separate parameters, so that we can obtain:

$$
\begin{aligned}
& \frac{\partial}{\partial x}\left(\frac{D}{G_{\text {core }} c} \nabla^{2} \omega-\omega-w\right)+\frac{\partial}{\partial y}\left[\frac{D\left(1-v_{f}\right)}{2 G_{\text {core }} c} \nabla^{2} f-f\right]=0 \\
& \frac{\partial}{\partial y}\left(\frac{D}{G_{\text {core }} c} \nabla^{2} \omega-\omega-w\right)-\frac{\partial}{\partial x}\left[\frac{D\left(1-v_{f}\right)}{2 G_{\text {core }} c} \nabla^{2} f-f\right]=0
\end{aligned}
$$

$$
\begin{aligned}
& \frac{D\left(1-v_{f}\right)}{2 G_{\text {core }} c} \nabla^{2} f-f+i\left(\frac{D}{G_{\text {core }} c} \nabla^{2} \omega-\omega-w\right) \\
& =F(x+i y)
\end{aligned}
$$

The corresponding homogeneous equations are:

$$
\begin{aligned}
& \frac{D\left(1-v_{f}\right)}{2 G_{\text {core }} c} \nabla^{2} f-f=0 \\
& \frac{D}{G_{\text {core }} c} \nabla^{2} \omega-\omega-w=0
\end{aligned}
$$

The complete solution is

$$
\begin{aligned}
& w=\frac{D}{G_{\text {core }} c} \nabla^{2} \omega-\omega \\
& D \nabla^{4} \omega=-q-2 t \times\left(\frac{D}{G_{\text {core }} c} \nabla^{2} \omega-\omega\right) \\
& \times\left(\sigma_{x 0} \frac{\partial^{2}}{\partial x^{2}}+\sigma_{y 0} \frac{\partial^{2}}{\partial y^{2}}+2 \tau_{x y 0} \frac{\partial^{2}}{\partial x \partial y}\right)
\end{aligned}
$$

\subsection{The single triangle progression method}

The exact solution for the bending of the thin rectangular panel under a uniformly distributed load with four edges simply supported is obtained by the single triangle progression method. The vertical displacement can be expressed:

$$
w(x, y)=\sum_{m=1}^{\infty} Y_{m}(y) \sin \frac{m \pi}{a} x
$$

Where $Y_{m}$ is an arbitrary function of $y$, and $\mathrm{m}$ is any positive integer.

$Y_{m}$ is selected which can be satisfied by the formula (20) and the boundary conditions, then: 


$$
\begin{aligned}
& \nabla^{4} w=\frac{q}{D} \\
& \frac{q}{D}=\frac{2}{a} \sum_{m=1}^{\infty}\left[\int_{0}^{a} \frac{q}{D} \sin \frac{m \pi x}{a} d x\right] \sin \frac{m \pi}{a} x \\
& Y_{m}^{(4)}(y)-2\left(\frac{m \pi}{a}\right)^{2} Y_{m}^{(2)}(y)+\left(\frac{m \pi}{a}\right)^{4} Y_{m}(y) \\
& =\frac{2}{a D} \sum_{m=1}^{\infty} \int_{0}^{a} q \sin \frac{m \pi x}{a} d x
\end{aligned}
$$

Whatever $\mathrm{x}$ takes on an arbitrary value, the formula (23) can be satisfied, we have

$$
\begin{aligned}
& Y_{m}=A_{m} \operatorname{ch} \frac{m \pi y}{a}+B_{m} \frac{m \pi y}{a} \operatorname{sh} \frac{m \pi y}{a} \\
& +C_{m} \operatorname{sh} \frac{m \pi y}{a}+D_{m} \frac{m \pi y}{a} \operatorname{ch} \frac{m \pi y}{a}+f_{m}(y) \\
& w=\sum_{m=1}^{\infty}\left(\begin{array}{l}
A_{m} \operatorname{ch} \frac{m \pi y}{a}+B_{m} \frac{m \pi y}{a} \operatorname{sh} \frac{m \pi y}{a} \\
+C_{m} \operatorname{sh} \frac{m \pi y}{a}+ \\
D_{m} \frac{m \pi y}{a} \operatorname{ch} \frac{m \pi y}{a}+f_{m}(y)
\end{array}\right) \sin \frac{m \pi}{a} x
\end{aligned}
$$

Where $f_{m(y)}$ is any of particular solutions and $A_{m}, B_{m}, C_{m}, D_{m}$ are constants.

$$
f_{m}(y)=\frac{2 q a^{4}}{\pi^{5} D m^{5}}(1-\cos m \pi)
$$

Because the panel is symmetry related to the $x$ direction, the formula (24) retains the even terms of $y$. Thus, $C_{m}=0$, $D_{m}=0$. By substituting the formula (23) into the formula (19), the final deflection formula is obtained:

$$
\begin{aligned}
& w=\sum_{m=1,3,5 \cdots}^{\infty}\left(\begin{array}{l}
\frac{2 q a^{4}}{\pi^{5} D m^{5}}(1-\cos m \pi) \\
+A_{m} \operatorname{ch} \frac{m \pi y}{a} \\
+B_{m} \frac{m \pi y}{a} \operatorname{sh} \frac{m \pi y}{a}
\end{array}\right) \sin \frac{m \pi x}{a} \\
& w=\frac{5 q a^{4} b}{384 D}+\frac{q a^{2} b}{8 U}
\end{aligned}
$$

Rectangular sandwich panel of equal surface layers is analyzed with four simply supported edges under uniformly transverse load $q$. The size of the panel is $a \times b$. The load on sandwich panel can be obtained:

$$
P=q a b
$$

When the material and thickness of sandwich panel are same, the deflection calculation formula at mid-span can be derived under uniform load:

$$
w=\frac{k_{b} P a^{3}}{D}+\frac{k_{s} P a}{U}
$$

The first term on the right-hand side of Eq.(29) is the deflection due to bending, and the second term is the deflection due to shear. $k_{b}$ and $k_{s}$ are the bending and shear deflection coefficients, respectively. The values of both $k_{b}$ and $k_{s}$ depend on the loading and boundary conditions. The bending and shear deflection coefficients under different loading and boundary conditions can be obtained. $k_{b}$ and $k_{s}$ are $5 / 384$ and $1 / 8$, respectively.

According to the theory of combination beam, formula for translation of axis and ASTM C-393, the formula of moment of inertia per unit width sandwich panel can be obtained[13-17]:

Where e $\mathrm{t} 1, \mathrm{t} 2$ are surface layer thicknesses and tc is core thickness.

$$
I=\frac{\left(t_{1}^{3}+t_{2}^{3}\right)}{12}+\frac{t_{1} \times\left(t_{c}+t_{2}\right)^{2}+t_{2} \times\left(t_{c}+t_{1}\right)^{2}}{4}
$$


By substitution of the size of CSIPs as shown in Fig.1. into the formula (30),we can obtain the flexural and shear rigidities:

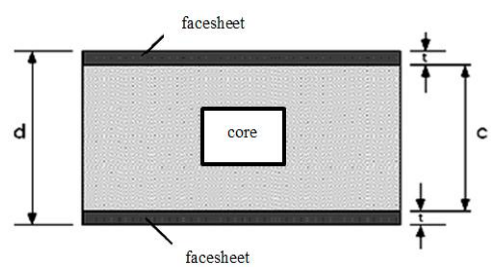

Fig. 1. Schematic figure for the CSIPs

$$
\begin{aligned}
& t_{c}=c \\
& t_{1}=t_{2}=\mathrm{t}=\frac{d-c}{2} \\
& I=\frac{\left(d^{3}-c^{3}\right) b}{12} \\
& D=E_{f} I\left(1-v_{x y}^{2}\right)=\frac{E_{f}\left(d^{3}-c^{3}\right) b}{12}\left(1-v_{x y}^{2}\right)
\end{aligned}
$$

Where $d_{\text {eff }}$ should be used to instead of $d$ in the formula (31) and (32) when determining the flexural and shear rigidities[9]:

$$
\begin{aligned}
& d_{e f f}=t\left(1+\frac{c}{d}\right)+0.3 \frac{c^{2}}{d} \\
& c_{e f f}=d_{e f f}-2 t
\end{aligned}
$$

Using the same way, the shear rigidity can be obtained[8-9]:

$$
\begin{aligned}
& U=\frac{G(d+c)^{2} b}{4 c} \\
& \Delta=\frac{k_{b} P L^{3}}{D}+\frac{k_{S} P L}{U}
\end{aligned}
$$

Where $b$ and $L$ are the width and length of the panel, and $\Delta$ is the displacement of the panel.

According to ASTM C-393, ultimate flexural bearing capacity of CSIPs is determined from Eqs.(34) [6-9]:

$$
q_{c r}=\frac{[\sigma]}{\frac{L^{2}}{8 c t}+\frac{L^{2}}{8 c^{2}}+\frac{E_{f} t}{2 c G_{\text {core }}}}-\frac{G_{m}}{b L}
$$

\section{Analysis of an example}

CSIPs are made up the glass fiber reinforced composites (FRP) and expanded polyethylene (EPS) foam. The thickness is $103 \mathrm{~mm}$ with a surface layer thickness of $1.5 \mathrm{~mm}$ and a core thickness of $100 \mathrm{~mm}$. Material properties of CSIPs are listed in Table 1, 2 .

\subsection{Theoretical calculations}

By substitution of each parameter of CSIPs into the deducing procedure, the deformation and bearing capacity of CSIPs are obtained as following: 
Table 1. Material properties of FRP

\begin{tabular}{|c|c|}
\hline Project & Numerical \\
\hline Thickness of face sheet $(\mathrm{t})$ & $1.5 \mathrm{~mm}$ \\
\hline Density $\left(\rho_{f}\right)$ & $980 \mathrm{~kg} / \mathrm{m}^{3}$ \\
\hline Glass fiber content & $70 \%$ \\
\hline $\begin{array}{c}\text { Modulus of elasticity along } \\
\text { length }\left(E_{x}\right)\end{array}$ & $15,169 \mathrm{MPa}$ \\
\hline $\begin{array}{c}\text { Modulus of elasticity along } \\
\text { width }\left(E_{y}\right)\end{array}$ & $15,169 \mathrm{MPa}$ \\
\hline $\begin{array}{c}\text { Modulus of elasticity along } \\
\text { depth }\left(E_{z}\right)\end{array}$ & $1050 \mathrm{MPa}$ \\
\hline Poisson's ratio $\left(v_{x y}, v_{y z}, v_{x z}\right)$ & $0.11,0.22,0.22$ \\
\hline Shear modulus $\left(G_{x y}, G_{y z}, G_{x z}\right)$ & $1800 \mathrm{MPa}, 1800 \mathrm{MPa}, 750 \mathrm{MPa}$ \\
\hline Tensile strength & $690 \mathrm{MPa}$ \\
\hline Compassion strength & $317 \mathrm{MPa}$ \\
\hline
\end{tabular}

Table 2. Material properties of EPS

\begin{tabular}{|c|c|}
\hline Project & Numerical \\
\hline Thickness of core $(t)$ & $100 \mathrm{~mm}$ \\
\hline Density $\left(\rho_{f}\right)$ & $16 \mathrm{~kg} / \mathrm{m}^{3}$ \\
\hline Modulus of elasticity $\left(E_{c}\right)$ & $1.2-1.5 \mathrm{MPa}$ \\
\hline Poisson's ratio $(v)$ & 0.25 \\
\hline Shear modulus $(G)$ & $1.9-2.2 \mathrm{MPa}$ \\
\hline Tensile strength & $0.11-0.14 \mathrm{MPa}$ \\
\hline Compassion strength & $0.07-0.1 \mathrm{MPa}$ \\
\hline
\end{tabular}

$$
\begin{aligned}
& d_{e f f}=t\left(1+\frac{c}{d}\right)+0.3 \frac{c^{2}}{d} \\
& =1.5 \times\left(1+\frac{100}{103}\right)+0.3 \times \frac{100^{2}}{103} \\
& =32.0825 \mathrm{~mm} \\
& c_{\text {eff }}=d_{\text {eff }}-2 t=32.0825-3 \\
& =29.0825 \mathrm{~mm} \\
& D=E_{f} I=\frac{E_{f}\left(d^{3}-c^{3}\right) b}{12}\left(1-v_{x y}^{2}\right) \\
& =\frac{15169 \times\left(32.0825^{3}-29.0825^{3}\right) \times 1000}{12} \times\left(1-0.11^{2}\right) \\
& =1.0520 \times 10^{10} \mathrm{~N} \cdot \mathrm{mm}^{2} \\
& U=\frac{G_{\text {core }}(d+c)^{2} b}{4 c}=\frac{2.2 \times(32.0825+29.0825)^{2} \times 1000}{4 \times 29.0825} \\
& =7.0752 \times 10^{4} \mathrm{~N} \\
& \Delta=\frac{k_{b} P L^{3}}{D}+\frac{k_{S} P L}{U} \\
& =\frac{5 \times 1000 \times 1000^{3}}{1.0520 \times 10^{10} \times 384}+\frac{1000 \times 1000}{7.0752 \times 10^{4} \times 8} \\
& =3.0045 \mathrm{~mm} \\
& q_{c r}=\frac{[\sigma]}{\frac{a^{2}}{8 c t}+\frac{a^{2}}{8 c^{2}}+\frac{E_{f} t}{2 c G_{\text {core }}}}-\frac{G_{m}}{a b}
\end{aligned}
$$




$=\frac{690}{\frac{1000^{2}}{8 \times 29.0825 \times 1.5}+\frac{1000^{2}}{8 \times 29.0825^{2}}+\frac{15169 \times 1.5}{2 \times 29.0825 \times 2.2}}$
$-82 \quad=122.3821 \mathrm{KN} / \mathrm{m}^{2}$

\subsection{Finite element simulation}

The universal finite element program-ANSYS12.0 was employed to establish model and analysis using 8-node threedimensional entity unit element (SILOD45). Due to bond together, glass fiber enhanced composite board and polystyrene foam has no relative movement model in sandwich panel. In this paper, the panel is simplified as ideal elastic material.

$u_{x}, u_{y}, u_{z}$ for all nodes in the edge of the sandwich panel carry on the restraint. In the static analysis, the uniform load of $1 \mathrm{kN} / \mathrm{m}^{2}$ on the sandwich panel is applied in the model. The finite element analysis model is shown in Fig.2.

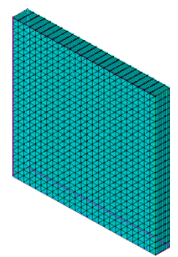

Fig. 2. Finite element model of CSIPs

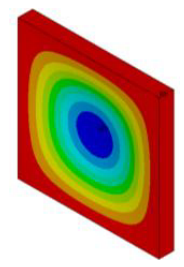

Fig. 3. Displacement of C

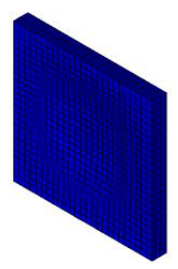

Fig. 4. The first bucking mode of CSIPs panel

The results of the finite element static analysis are shown in Fig.3. The maximum displacement value is located in the middle of the span, which is $3.1024 \mathrm{~mm}$. The results are in good agreement with those of the theoretical analysis. Eigenvalue buckling analysis was carried out on the model to obtain the buckling mode and the characteristic value of the coefficient, as shown in Fig.4. The first eigenvalue coefficient of Sandwich panel is 21.117, which is slightly smaller. It is caused mainly by the low shear strength of the core panel.

\subsection{Model tests}

The three same specimens were designed and made whose material are made up the glass fiber reinforced composites(FRP), expanded polyethylene (EPS) foam and a hot-melt thermoplastic spray adhesive. The glass fiber reinforced composite panel and polyethylene foam panel are cut into the length $1000 \mathrm{~mm}$ and breath $1000 \mathrm{~mm}$ panel using cutting tools. Applying evenly thermoplastic spray adhesive to the FRP, then placing it on the polyethylene foam board and bringing pressure to bear on it. The specimen after curing was shown in Fig.5. 
In loading process of experiments, firstly, the wires of the pressure sensor on the self- balancing anti force frame are connected with the equipment and the computer, and opening the test software. Then placing the specimen on the support and simulating the whole process from loading to failure. The calculation results of displacement and the critical load of the specimen are automatically recorded by the sensor. Experimental loading conditions are consistent with the boundary condition of the finite element model.

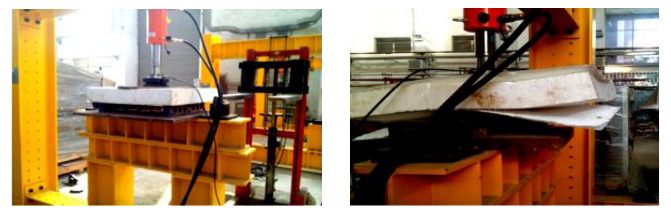

Fig. 5. The testing specimen and the loading equipment Fig. 6. Failure pattern of the specimen

With the increase of load, specimens made a slight sound. We cannot find significant change with the naked eye observation. The displacement of the mid-span increased with the increase of load; in loading process of experiments, specimens made "crack" sound and bended down into arc after observation; at a later stage of loading, obvious tearing sound can be heard and panels on the surface warped. Now it was found that core board and the edges of the upper layer appeared slight debonding phenomenon; with the further increase of load, the core and the lower layer bonding edge also appeared slight debonding phenomenon, the failure area extended constantly to the center and bearing capacity suddenly dropped, specimen failure is shown in Fig.6.

\section{Conclusion}

(1) A new type of sandwich panel, namely CSIPs (Composition Structural Insulated Panels), was proposed in this paper. CSIPs are made of low-cost orthotropic thermoplastic glass/polypropylene (glass-PP) laminate as face sheets and expanded polyethylene (EPS) foam as a core and are bonded together through a hot-melt thermoplastic spray adhesive.

(2) CSIPs have the advantages of light weight, high strength, better corrosion-resistant, high durability, energy conservation and environmental protection, low maintenance cost. It is also provided with features of convenient transportation and construction, especially the production methods of industrialization and assembled on site can greatly shorten the building cycle.

(3) Due to the relatively low elastic modulus of the FRP in the composite sandwich panel, the stiffness of the structure is small. Therefore, it is necessary to use the large deflection theory to analyze.

(4) Theoretical calculation, finite element simulation and experimental results are consistent. It is showed that the calculating formula of deformation and bearing capacity of CSIPs are correct.

\section{References}

1. J.F. Wu, F.H. Xu, D. Gao. Vibration isolation performance of precompression multi-layer calcium plastic corrugated board, Packaging Engineering 19,27(2015).

2. S.G. Wang, L.Zhang, D.S. Du, et al. Full scale shaking table tests on frame structure with new SIP filling wallboard, Journal of vibration and shock 18,100(2015).

3. S.G. Wang, T. Wu, W.Q. Liu, et al. The basic properties test of new SIP wall panel, New Materials 40, 72(2013).

4. U. Nasim, W.F Du. New thin shells made of composite structural insulated panels, Journal of Reinforced Plastics and Composites 33, 1954(2014).

5. A. Vaidya, N. Uddin, U. Vaidya. Design and analysis of composite structural insulated panels (CSIPs) for exterior wall applications, Journal of Composite Construction 28, 1587(2010).

6. M. Mousa, N. Uddin. Structural behavior and modeling of full-scale composite structural insulated wall panels, Journal of Engineering Structures 41, 320(2012).

7. M. Mousa, N. Uddin. Debonding of composites structural insulated sandwich panels, Journal of Reinforced Plastics and Composites 29, 3380(2010).

8. W.F. Du, N. Uddin. Behavior and connection of new CSIPs thin shell structures// Proceeding of 15 National Conference on Advances in Modern Structures Kaifeng, China, 483 (2015).

9. X.Y. Wang, F.B. Yang, J.C. Zeng. The design principle and its application of sandwich structural composites. Beijing: Chemical industry press, 2(2007). 